\title{
Editor's note:
}

In the era of personalized medicine, a critical appraisal new developments and controversies are essential in order to derived tailored approaches. In addition to its educative aspect, we expect these discussions to help younger researchers to refine their own research strategies.

\section{Ki-67 expression in pulmonary tumors - reply}

\author{
Mark Kriegsmann, Arne Warth \\ Institute of Pathology, Heidelberg University, Heidelberg, Germany \\ Correspondence to: Arne Warth, MD. Institute of Pathology, Heidelberg University, INF 224, D-69120 Heidelberg, Germany. \\ Email: arne.warth@med.uni-heidelberg.de.
}

Submitted Sep 13, 2016. Accepted for publication Sep 28, 2016.

doi: $10.21037 /$ tlcr.2016.10.12

View this article at: http://dx.doi.org/10.21037/tlcr.2016.10.12

First of all, we would like to congratulate Professor Chirieac for his excellent discussion on proliferation assessment in lung tumors. Taken together, he concludes that assessment of proliferation is important for the prognosis of non-small cell lung cancer (NSCLC), prediction of brain metastases in patients with lung adenocarcinoma, and the diagnosis, classification and prognosis of pulmonary neuroendocrine tumors but is subjective, has a poor reproducibility, and is depended on laboratory techniques-which is more or less in line with our opinion as well as our own data (1-4). Furthermore, he states that $\mathrm{Ki}-67$ is a widely accepted method for the evaluation of the proliferative activity and emphasizes its role in clinical practice for neuroendocrine tumors of the gastrointestinal tract. We fully agree on this notion.

In 2008, Professor Chirieac and colleagues investigated associations between immunohistochemical markers and the development of brain metastases in patients with NSCLC and found that a high Ki-67 Index was associated with an increased risk of developing brain metastases (5). Although the patient size $(n=21)$ was rather small and additional studies on larger cohorts are clearly needed to validate these findings, this study is an excellent example to underline the potential of Ki-67 to predict clinical outcomes.

Furthermore, it is pointed out that mitoses may be difficult to assess in biopsies, that the assessment is time consuming and that it might sometimes be challenging to distinguish mitotic figures from apoptotic cells. Thus, it is intelligible that a higher intra- and interobserver conformity is required (6). We agree that the assessment of a nuclear stain is easier to evaluate than mitoses. This also explains why certain laboratories seek to replace mitotic counting by Ki-67 staining in specific constellations (7). Professor Chirieac further recommends the use of Ki-67 for the diagnosis of all pulmonary neuroendocrine tumors, which is a comprehensible argument based on the current literature and specifically helpful in the setting of cytology or biopsy specimens in order not to misdiagnose e.g., a SCLC as a carcinoid or vice versa (8). However, officially $\mathrm{Ki}-67$ is currently not recommended since specific cut-offs for lung neuroendocrine tumors are not established at this time (9). Despite these current shortcomings there is no doubt that $\mathrm{Ki}-67$ is a valuable component to be integrated in future diagnostic algorithms for lung neuroendocrine tumors.

Although the prognostic and predictive potential of proliferation assessment in NSCLC is obvious, a significant step forward can only be achieved by harmonized international efforts to standardize respective methods. In this regard, the assessment of an immunohistochemical marker like $\mathrm{Ki}-67$ is a suitable candidate since the new generation of immunohistochemical staining platforms as well as the better standardization of protocols or even the use of commercially available kits allows for a high reproducibility among different laboratories, as most recently demonstrated for different clones of programmed death-ligand 1 (PD-L1) (10). Moreover, imaging analysis 
software for virtual pathology may also contribute to a more standardized evaluation of Ki-67 in the future. However, one important caveat might be that essential diagnostic criteria should usually be applicable worldwide. In this regard, counting mitotic figures in hematoxylin and eosin stained tissue sections is an easy and inexpensive way to assess proliferation whereas immunohistochemical analyses and especially also digital image analyses are often only available in developed countries or specialized institutes. This might be considered an argument against $\mathrm{Ki}-67$ as a mandatory diagnostic criterion. However, despite the consistent increase in workload for pathologists (11), application of standardized and reproducible immunohistochemical and molecular parameters for diagnosis, prognosis, and prediction undoubtedly propelled the significant progress made in the field of tumor subtyping over the last decade. Integration of a clinically meaningful proliferation assessment is now one of the future challenges in this context.

\section{Acknowledgements}

None.

\section{Footnote}

Conflicts of Interest: The authors have no conflicts of interest to declare.

Comment on: Chirieac LR. Ki-67 expression in pulmonary tumors. Transl Lung Cancer Res 2016;5:547-51.

\section{References}

1. Warth A, Cortis J, Soltermann A, et al. Tumour cell proliferation (Ki-67) in non-small cell lung cancer: a critical reappraisal of its prognostic role. Br J Cancer 2014;111:1222-9.

Cite this article as: Kriegsmann M, Warth A. Ki-67 expression in pulmonary tumors-reply. Transl Lung Cancer Res 2016;5(5):552-553. doi: 10.21037/tlcr.2016.10.12
2. Warth A, Fink L, Fisseler-Eckhoff A, et al. Interobserver agreement of proliferation index (Ki-67) outperforms mitotic count in pulmonary carcinoids. Virchows Arch 2013;462:507-13.

3. Zahel T, Krysa S, Herpel E, et al. Phenotyping of pulmonary carcinoids and a Ki-67-based grading approach. Virchows Arch 2012;460:299-308.

4. Warth A, Muley T, Harms A, et al. Clinical Relevance of Different Papillary Growth Patterns of Pulmonary Adenocarcinoma. Am J Surg Pathol 2016;40:818-26.

5. Saad AG, Yeap BY, Thunnissen FB, et al. Immunohistochemical markers associated with brain metastases in patients with nonsmall cell lung carcinoma. Cancer 2008;113:2129-38.

6. Garbe C, Eigentler TK, Bauer J, et al. Mitotic rate in primary melanoma: interobserver and intraobserver reliability, analyzed using H\&amp;E sections and immunohistochemistry. J Dtsch Dermatol Ges 2016;14:910-5.

7. Christgen M, Länger F, Kreipe H. Histological grading of breast cancer. Pathologe 2016;37:328-36.

8. Aslan DL, Gulbahce HE, Pambuccian SE, et al. Ki67 immunoreactivity in the differential diagnosis of pulmonary neuroendocrine neoplasms in specimens with extensive crush artifact. Am J Clin Pathol 2005;123:874-8.

9. Pelosi G, Rindi G, Travis WD, et al. Ki-67 antigen in lung neuroendocrine tumors: unraveling a role in clinical practice. J Thorac Oncol 2014;9:273-84.

10. Scheel AH, Dietel M, Heukamp LC, et al. Harmonized PD-L1 immunohistochemistry for pulmonary squamous-cell and adenocarcinomas. Mod Pathol 2016;29:1165-72.

11. Warth A, Stenzinger A, Andrulis M, et al. Individualized medicine and demographic change as determining workload factors in pathology: quo vadis? Virchows Arch 2016;468:101-8. 